\title{
Open Source Wireless Sensor Network Classroom Monitoring System
}

\author{
$1^{\text {st }}$ Muladi \\ Departement of Electrical Engineering, \\ Faculty of Engineering, Universitas Negeri Malang, \\ Jawa Timur, Indonesia \\ muladi@um.ac.id \\ $2^{\text {nd }}$ Marji \\ Departement of Mechanical Engineering, \\ Faculty of Engineering, Universitas Negeri Malang, \\ Jawa Timur, Indonesia \\ teknikmesin@um.ac.id
}

\author{
$3^{\text {rd }}$ Heru Wayhu \\ Departement of Mechanical Engineering, \\ Faculty of Engineering, Universitas Negeri Malang, \\ Jawa Timur, Indonesia \\ heru_wh@um.ac.id \\ $4^{\text {th }}$ Samsul Hiday at \\ Departement of Mechanical Engineering, \\ Faculty of Engineering, Universitas Negeri Malang, \\ Jawa Timur, Indonesia \\ samsulhdy@yahoo.com
}

\begin{abstract}
One of the factors that affect student achievement is the condition of the study. The intensity of lighting, air composition, humidity, and temperature in the room is the main factors that determine the health and comfort of the students. Changes in weather and climate, interior design, and the use of instructional media will affect these factors. The use of electronic devices such as air conditioners (AC) to improve the comfortness of the room can be turned into a source of disruption to health if it is not accompanied by the air quality control. This research aims to design and build a monitoring the condition of the classroom for providing comfortness using open source wireless sensor networks (WSN) and web-based user interface. The research is conducted in the research development methode manner. Hardware using WifiBee as a major component equipped WSN sensors and a data connection through wifi. Software design is to build a web server that consists of Apache, PHP and MySQL. Both hardware dan software is developed using open source. The results show that open source WSN can be used for monitoring the condition of the classroom. Measurement data can be stored and accessed through a wireless network.
\end{abstract}

Keywords -IoT, open source, classroom monitoring

\section{INTRODUCTION}

Wireless sensor network or in a lot of literature called Wireless Sensor Network (WSN) is a network that connects devices such as sensor nodes, routers and node sink [1]. Principles of Wireless Sensor Network (WSN) or WSN uses one or more sensor devices that are connected wirelessly to form a network and is used for various purposes such as environmental monitoring [2], weather [3], temperature [4], volcano activity [5], monitoring and control activities in the field agriculture [6], and so on which will be more easily done through a wireless network.

The works of WSN is a sensor change analog data into digital data that is subsequently sent to a node through a communication medium which is used as Bluetooth, infrared or wifi. Each node in the WSN is equipped with a radio transceiver or receiver nodes as well as other supporting devices. So that the WSN is also known as a system consisting of a large number of low-cost sensors that are small and scattered in a very large area with a single node reservoir to collect the results of the reading process other sensor nodes [7].

Nodes that are commonly used for the application of ZigBee WSN is, which uses radio frequency as the communication medium because ZigBee has become one of the standards for WSN [8]. An alternative that can be used to build a WSN is WifiBee that use wifi as a medium of communication so as to take advantage of local wireless network infrastructure that already exists and has been widely used as in schools, offices, and public facilities for the purpose of environmental monitoring such as temperature and moisture on the scope of a particular place.

This research will be explained through the stages in building a Wireless Sensor Network using WifiBee that ranging from configuration to connect to the server over the wireless network and display the data from the sensor to the browser. The server is conFig.d as a system to process the data from the sensors to as web-based monitoring.

\section{HARDW ARE DESIGN}

In designing the hardware, there are some devices that are used as WifiBee, Grove - XBee Carrier, and Grove Temperature \& Humidity Sensor. Fig. 1 shows the block diagram of the system. WifiBee plays two functions as microcontroller and wireless interface. The wireless network connection is build using IEEE $802.11 \mathrm{~b}$ Standard.

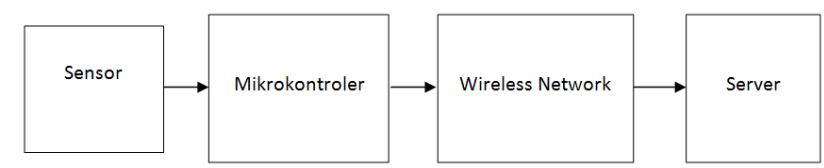

Fig. 1. Block Diagram System

The system consists of 4 blocks is a block in which the sensor is used to measure temperature and humidity, the 
second block is a microcontroller that of the WifiBee function to read data from the sensor and then create a web page as a place where the measurement sensor the data displayed, the third block is the network which serves as a medium of wireless data traffic between the server and WifiBee, and the latter is a server that functions to process the data from the database and display it in the form of tables and graphs for monitoring purposes.

\section{A. WifiBee Modul}

WifiBee is a standalone (microcontroller unit (MCU) node that integrates an 8bit AVR MCU ATMega328P and Microchip IEEE $802.11 \mathrm{Wi}-\mathrm{Fi}$ transceiver module MRF24WB0MA. ATMega328P processor works on 16 $\mathrm{MHz}$ clock speed with low voltage supply at a range of 3 to 3,6 volts. A Xbee Carrier compatible socket is used to extend the connection with sensor boards and others. MCU ATMega328P could be directly programmed under the open source Arduino IDE when it plugged in through UART. For enabling communication capability to each other, the MCU is connected to the wifi module MRF24WB0MA using its SPI port. This wifi module supports low and high rate data transfer which tipically occurs in WSN and also enable security features using WEP, WPA-PSK, WPA2-PSK. The other pin of MCU is brought out to the 20 pins of $2.0 \mathrm{~mm}$ male pin headersis for conneting with sensor or actuator modules. The MCU is already Arduino Duemilanove bootloader preprogrammed. Arduino-compatible MCU board is one of the open-source platforms that easy and simple to use as a development board for prototyping the microcontroller-based electronic devices [13]. Fig. 2 shows the WifiBee Module.

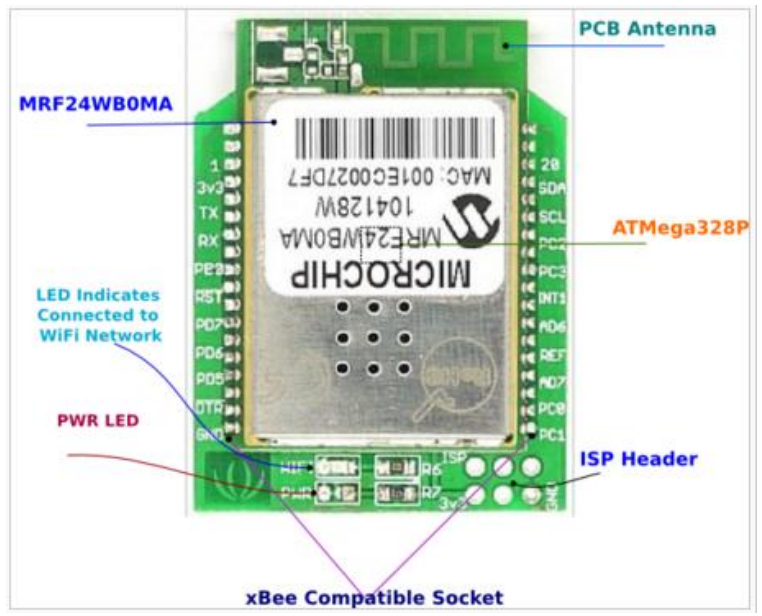

Fig. 2. WifiBee Modul [10]

\section{B. XBee Carrier}

XBee Carrier board is designed for basic series and the Bee Grove unit is compatible for the standalone node as RFBee, WifiBee, XBee (ZigBee) and Bluetooth Bee. This board as shown in Fig. 3 is given the power to use lithium batteries or via the USB cable [10]. This board comes with an $\mathrm{I} 2 \mathrm{C}$ port and two digital ports to connect the Bee with sensor or actuator. Mini USB socket is provided as an interface for programming using Arduino IDE. Two options for charging the board is available, external DC charging (power adapter or solar panel) and baterry connector.

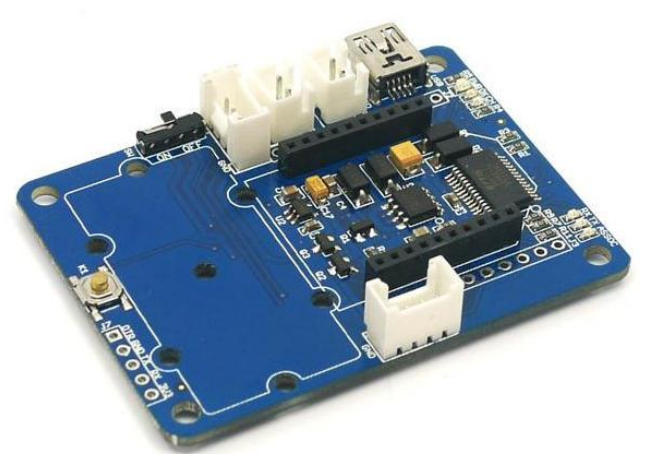

(a)

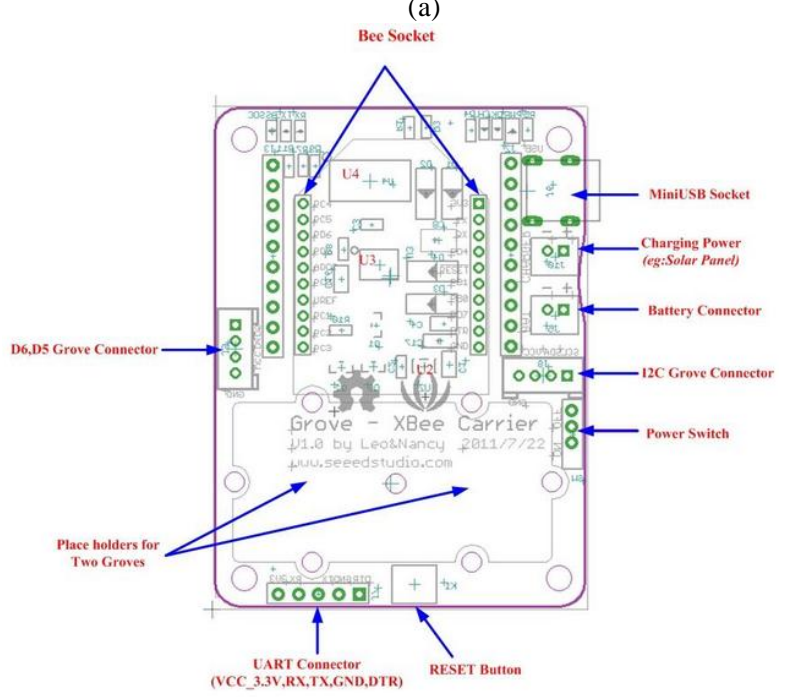

(b)

Fig. 3. XBee Carrier, (a) board, (b) interface conncection

\section{Temperature and Humidity Sensor}

Multifunctional sensors that can provide information on the temperature and humidity at the same time. DHT series module capable of sensing both temperature and humidity at the same time. The available series are DHT11 and DHT22. Individual sensor for temperature and humidity are available but requires more port of MCU. Sensor quality will define the accuracy of measurement therefore high quality ones are selected. Ordinary, high quality product can be obtained from proprietary technologies that ensure high reliability and long-term stability is excellent [11]. The dht11 sensor module is shown in Fig. 4.

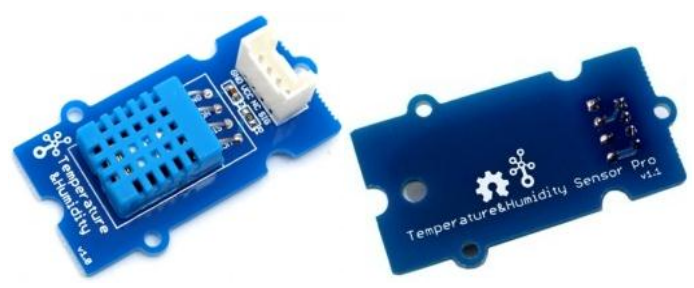

Fig. 4. Temperature and Humidity Sensor Modules

\section{Realization of Hardware}

WifiBee is a module and to use it to be screwed into the socket XBee Carrier. Carrier XBee connected to the PC using a USB cable as a medium of communication while uploading sketchyang also function as a resource for XBee Carrier when connected to the USB port on the PC, in addition to using a USB cable can also use the battery. All devices ranging from WifiBee, Grove - XBee Carrier, and Grove - Temperature \& Humidity Sensor has been designed 
for ease of use in such a way so as not to overly complicated in WSN applications. WifiBee is programmed using the Arduino IDE to upload a sketch to the microcontroller that is on WifiBee.

Fig. 5 shows the results of the entire circuit node that consists of a sensor, WifiBee and XBee Carrier which is connected using a USB cable with the power supply in the form of a Power Bank instead of the batteries. The goal is for the WSN simulation closer to reality its application, namely, compact, mobile, and wireless based.

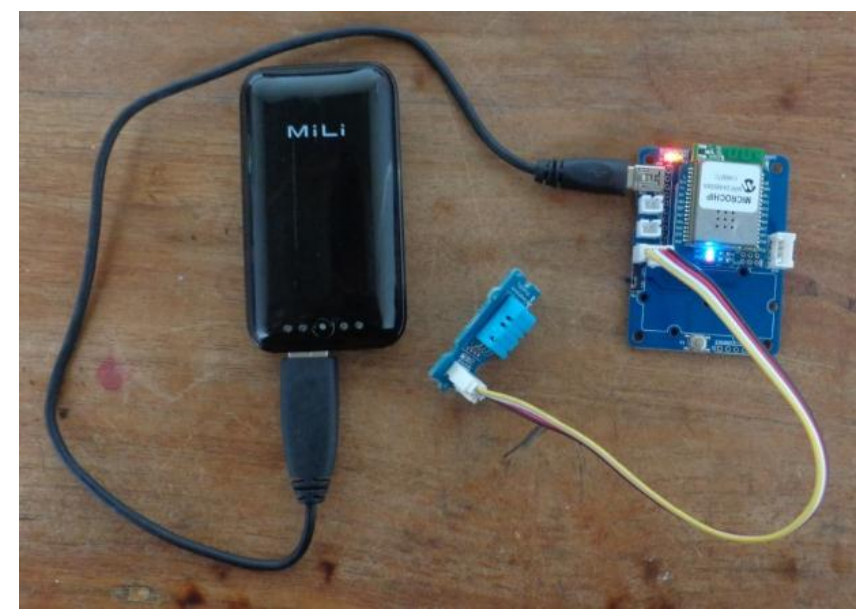

Fig. 5. Sensor Node

\section{SOFTWARE DESIGN}

Fig. 6 shows the workflow of the system was made to build the WSN and its monitoring.

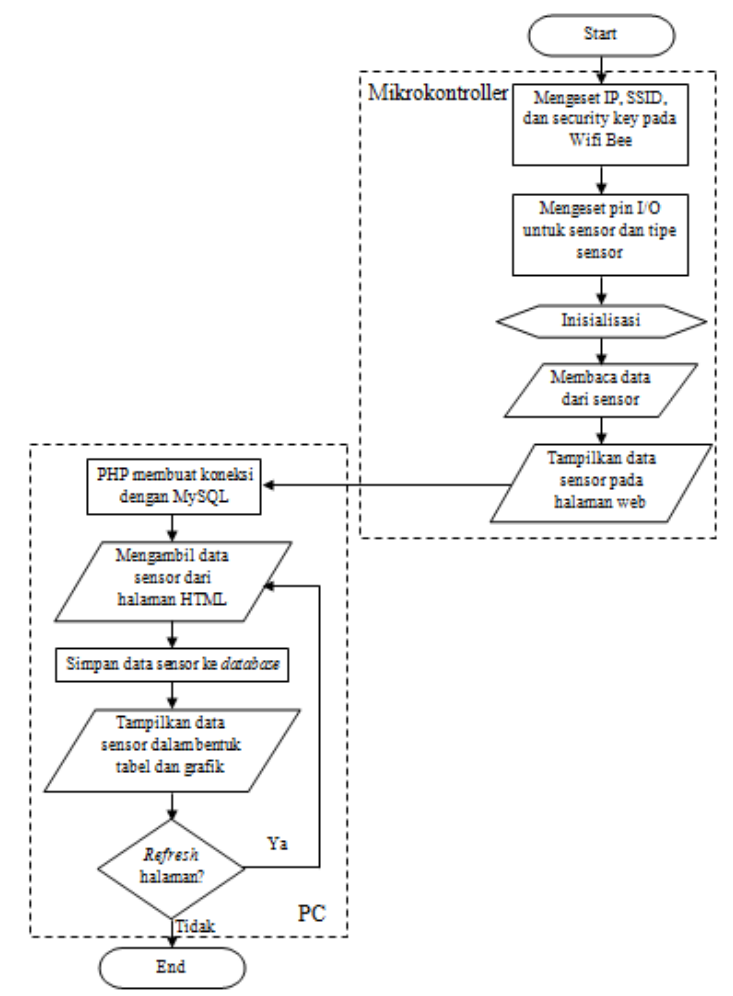

Fig. 6. Flowchart System

The flowchart above is the working principle of the system when the power switch is turned on and then upload the Arduino sketch the first time to set the IP address, SSID and security key on WifiBee and determine the I/O pins and the type of sensor used. After initializing WifiBee then start reading the data from the sensor. WifiBee builds html pages to display data that has been retrieved from the sensor readings. PHP pages take the content of the html page and convert it into a string and split into an array containing the values of temperature and humidity. Temperature and humidity values are stored in the database. Other PHP pages to retrieve data from the database to be displayed in the form of tables and other pages to create an array of monitoring the formation of the chart so that the page contains information tables and graphs of temperature and humidity measurements.

Testing the whole system is objected to determine whether the WSN WSN system which consists of the incorporation of software and hardware to run properly. Tests performed include testing the responsiveness and Wifi connectivity Bee by distance, testing is performed incrementally monitoring system with access to every configuration file that was created in sequence to produce the output as expected, as well as testing the entire WSN system conducted periodically to ensure the systems and tools function well as a simulation of a real WSN.

\section{RESULTS AND DISCUSSIONS}

The results of testing the whole system WSN shows that the system consisting of hardware and software that includes: Grove - Temperature \& Humidity Sensor which can measure temperature and humidity, WifiBee mounted on XBee Carrier can perform data readout from the sensor and displays the data on a web page, as well as servers that perform the capture, storage and display data from the database to work in accordance with the design and obtained the results as shown in Fig. 7, which shows the main page shows a graph of temperature monitoring at the same time in the last 10 measurements. The interface uses Bahasa Indonesia and can be adopted to English easily.

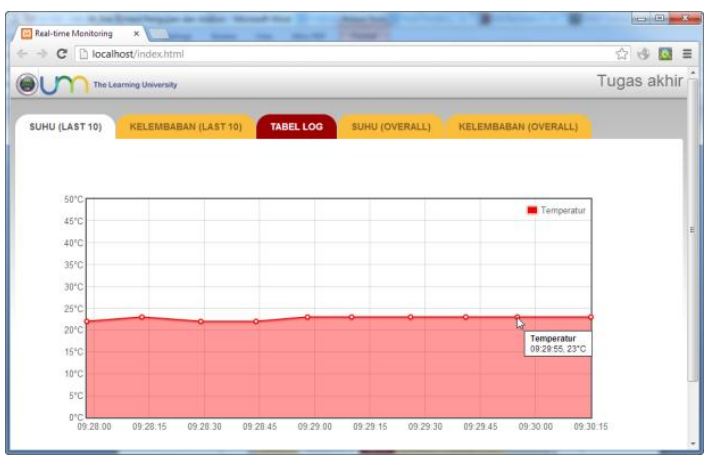

Fig. 7. Monitoring Webpage

The main page consists of 5 pieces tab with 4 tabs and 1 tab contains the graph contains a table of all of which can display data in real-time because the automatic refresh at defined intervals without having to refresh the main page as shown in Fig. 8.

On the first tab there is a dynamic chart that shows the results of temperature measurements in the last 10 measurements. The ordinat of the graph presents the measured temperature while the absis shows the time of measurement. The value of temperature is displaying dynamically following the result of measurement which has spreaded value in the range of $0-50$ degree centigrade. The sensors have calibrated prior measurement and the gap or error has compensated by the software. The measurement has taken place every 15 seconds and can be ajusted 
according to the requirement. The graph shows ten records of measurement while the previous results stored in the database for next another usage.

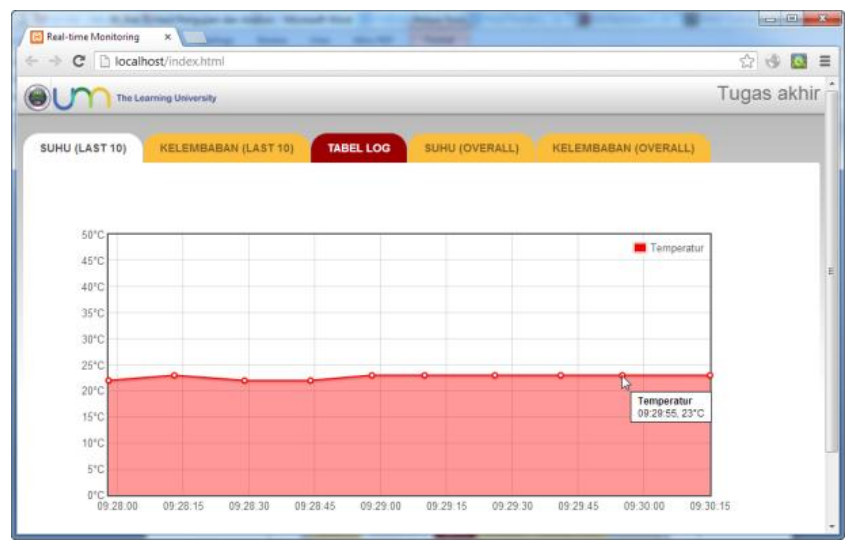

Fig. 8 Temperature Display tab

The second tab there is a dynamic chart that shows the results of measurements of moisture in the last 10 measurements as shown in Fig. 9. Similar to the first tab, the graph has a label Humidity, the absis shows time information by a margin of 15 seconds and ordinat shows a large moisture as measured by a margin of $5 \%$ with a range between $20 \%-90 \%$ according to the range that is able to be measured by the sensor. All of the measurement records are recorded in the database for future usage. The data can export to spreadsheet workbook for enabling numerical or statistical analysis and extended processing such as decission support system (DSS). Fig. 10 shows the data structure stored in the MYSQL database.

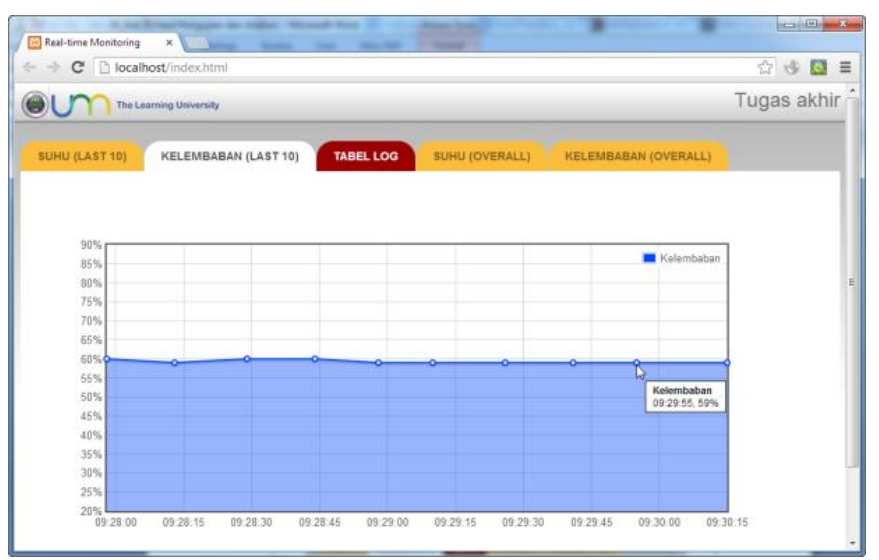

Fig. 9. Humidity Display tab

The third tab contains a dynamic table that shows the last 10 measurements are sorted based on the longest time until the latest time. The table consists of ID number, which shows the time when the data is stored. Temperature tabs show the measured temperature, as well as humidity which shows the value of moisture. The graph is shown in Fig. 11.

The fourth tab contains a dynamic chart that shows the overall temperature measurements obtained from the database with the same graph formats as in the first tab. The graph is shown in Fig. 11. And the last tab contains a dynamic graph in Fig. 12, which shows the overall moisture measurements obtained from the database with the same graphics formats as in the second tab.

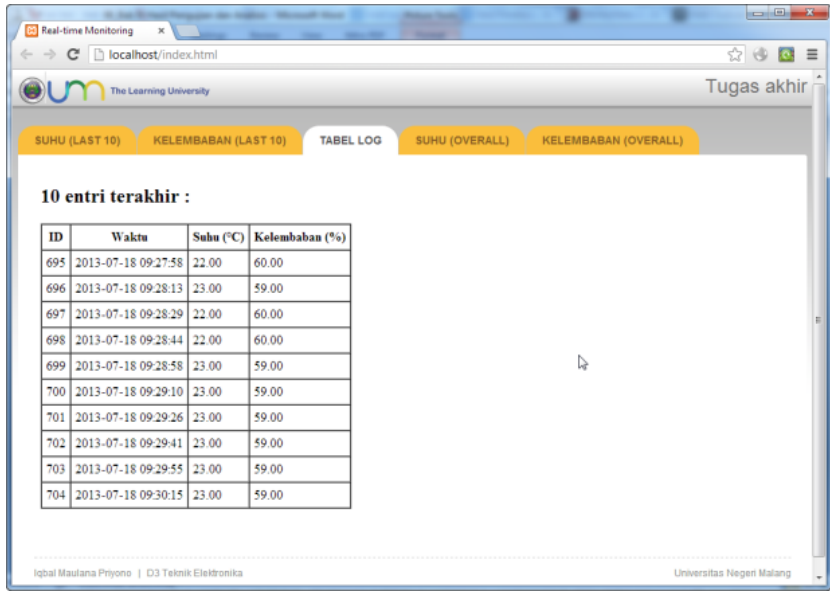

Fig. 10. Log Table Display tab

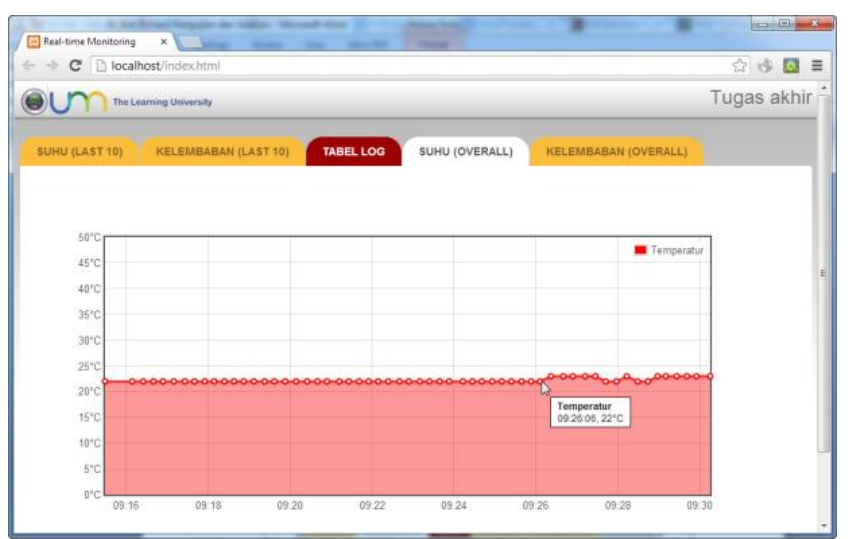

Fig. 11. Overall Temperature Display

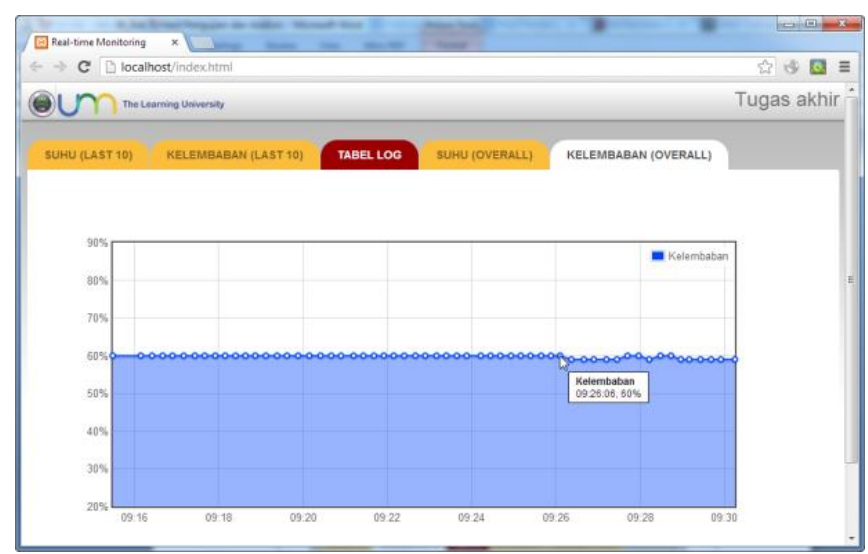

Fig. 12. Overall Humidity Display

\section{CONCLUSIONS}

Based on the test results some conclusions can be obtained as follows. To build a Wireless Sensor Network using WifiBee configuration is required on the web server and WifiBee that are connected to the network, Wi-Fi configuration Bee a way to add an Arduino sketch header WiServer in order to WifiBee has Wifi support such as IP addresses, security keys, and SSID can be changed as needed. Wi-Fi Configuration Bee in order to be able to read the data from the sensors to be sent to a web browser is to add a header to the sketch DHT11 Arduino IDE that is able to use the WifiBee specific functions to read the sensor data such as temperature and humidity. Implementation of Wireless Sensor Network using WifiBee associated with 
temperature and humidity sensors with various objectives, among others, as a thermometer to determine the temperature and humidity conditions in areas difficult to reach, a place that should not be frequented as server rooms, or places that require monitoring continuously. Using sensor data from the measurement results to be processed into a web-based monitoring system is to use MySQL as a database to store data from sensors, PHP to make a connection with a database, store and retrieve these data for monitoring the output in the form of tables and graphs that utilize jQuery.

To obtain better measurement results should use more precise sensors and has a faster response generated so that a more accurate data. For Wireless Sensor Network applications are actual, should be provided with the resources to meet the needs when used in a long time for example by using solar panel as a battery charger and a rechargeable battery so no need to replace the batteries periodically in a short time due to the limited power. Use more number of the sensor is able to measure many points in a wide area coverage.

\section{REFERENCES}

[1] Sohraby, K., Minoli, D., Znati, T. Wireless Sensor Network: Technology, Protocol, and Applications. New Jersey: Willy Interscience, 2007. pp. 15-37.
[2] Corke, P., Wark, T., Jurdak, R., Hu, W., Valencia, P., and Moore, D. "Environmental Wireless Sensor Network". Proc. of The IEEE, Vol. 98, No. 11, November 2010, pp. 1903-1917.

[3] Cholatip Yawut, C., and Kilaso, S. "A Wireless Sensor Network for Weather and Disaster Alarm Systems". 2011 International Conference on Information and Electronics Engineering (IPCSIT) vol. 6. 2011. IACSIT Press, Singapore.

[4] Mon, Y. J., Lin, C. M., and Rudas, I., J. "Wireless Sensor Network (WSN) Control for Indoor Temperature Monitoring". Acta Polytechnica Hungarica Vol. 9, No. 6, 2012.

[5] Allen, G., W., et. all. "Deploying a Wireless Sensor Network on an Active Volcano”. IEEE Internet Computing, March- April 2006.

[7] Keshtgary, M. and Deljoo, A. "An Efficient Wireless Sensor Network for Precision Agriculture". Canadian Journal of Multimedia and Wireless Net works, Vol. 3, No. 1, January 2012.

[8] Hartono, Hendro. "Wireless Sensor Network(WSN). (Online), (http://www.fararainside.net/2013/01/wireless-sensor-networkwsn.html), accessed July 31, 2013.

[9] ABI Research. 2012.850 Million IEEE 802.15.4 Chipsets to Ship in 2016, Despite Strong Competition from Bluetooth. (Online), (http://www.abiresearch.com/press/850-million-ieee-802154-chipsetsto-ship-in-2016-d), accessed July 31, 2013.

[10] SeeedWiki, 2011. WifiBee.(Online), (http://www.seeedstudio.com /wiki/Wifi_Bee), accessed February 20, 2016.

[11] SeeedWiki., 2013. Grove - XBee Carrier. (Online), (http://www.seeedstudio.com/wiki/Grove_-_XBee_Carrier), accessed February 20, 2016.

[12] SeeedWiki., 2013. Grove - Temperature \& Humidity Sensor. (Online), (http://www.seeedstudio.com/wiki/Grove_Temperature_and_Humidity_Sensor), accessed February 20, 2016.

[13] A. Javed. Building Arduino Projects for the Internet of Things: Experiments with Real-World Applications. Zurich: Apress. pp. 3-13. 